Artículo original

\title{
IMPACTO DE LA APLICACIÓN DEL PROCESO INMEDIATO EN LOS CASOS DE FLAGRANCIAEN EL PERÚ, 2016.
}

Impact of the application of the immediate process in cases of flagrancy in Peru, 2016.

\author{
José Felipe De La Barra Barrera ${ }^{1}$
}

\begin{abstract}
RESUMEN
El propósito del presente trabajo de investigación fue medir el impacto de la aplicación del proceso inmediato en los casos de flagrancia en el Perú durante el año 2016. Investigación de tipo aplicada, no experimental de corte transversal, de nivel descriptivo explicativa. Se consideró la información obtenida a través del cuestionario y la cédula de entrevista. Una vez finalizada la fase de análisis e interpretación de los resultados se precisó que la aplicación del proceso inmediato en los casos de flagrancia simplifica los actos procedimentales y de investigación que corroboren la existencia del delito y la responsabilidad penal del imputado, otorga mayor celeridad a los procesos y satisface oportunamente el daño sufrido por las víctimas, así como las expectativas ciudadanas.
\end{abstract}

\section{Palabras Clave: Flagrancia, Proceso Inmediato}

\section{ABSTRAC}

The purpose of this research was to measure the impact of the application of the immediate process in cases of flagrancy in Peru during the year 2016. Investigation of an applied type, not experimental cross-sectional, descriptive explanatory level. The information obtained through the questionnaire was considered the documentary analysis sheet; And the interview card. Once the phase of analysis and interpretation of the results was completed, it was pointed out that the application of the immediate process in cases of flagrancy simplifies procedural and investigative acts that corroborate the existence of the crime and the criminal responsibility of the accused, gives greater speed to the Processes and timely satisfaction of the damage suffered by the victims, as well as citizen expectations.

Keywords: Flagrance, Immediate Process

\section{INTRODUCCION}

Ante la creciente inseguridad que se vive en la capital y diversas localidades del país, el Poder

Doctor en Derecho, Universidad Privada de Tacna

Magistrado de la sala civil permanente de la corte suprema del Perú
Ejecutivo promulgó el Decreto Legislativo 1194 que regula el proceso inmediato en casos de flagrancia. El proceso inmediato es un proceso especial previsto en el Nuevo Código Procesal Penal (CPP) y procede en tres supuestos, cuando: a) la persona es sorprendida en flagrante delito, b) la persona confiesa el delito y c) hay suficiencia probatoria. En estos casos, el fiscal puede promover el proceso inmediato, dejando de lado el proceso común. (Sánchez, 2016). Con el Decreto Legislativo 1194 sobre flagrancia delictiva y proceso inmediato, se busca dar una rápida respuesta de justicia a una demanda social de seguridad frente al delito. El plan piloto, si bien es cierto tuvo ciertos problemas para su ejecución, no solo en el aspecto operativo presupuesto e infraestructura- en razón que todo proyecto requiere de un presupuesto para su implementación y ejecución, sino también en cuestión de recursos humanos -jueces, fiscales y abogados defensores propios de los órganos de flagrancia. Estos resultados animaron al Poder Ejecutivo a promulgar el Decreto Legislativo $\mathrm{N}^{\circ} 1194$. Frente al panorama advertido corresponde adoptar medidas correctivas necesarias y pertinentes y nos planteamos la pregunta ¿Cuál es el impacto de la aplicación del proceso inmediato en los casos de flagrancia en el Perú en el 2016?. El presente trabajo permitirá precisar la doctrina sobre el impacto de la aplicación del proceso descrito y a través del derecho comparado poder analizar las diferentes normas que permitan dar alcances de solución a la problemática citada.

Desde un punto de vista empírico, la flagrancia existe, es evidente y prácticamente indiscutible. Al aplicar la nueva estrategia procesal se genera una mayor sensación de seguridad en la ciudadania(Rebolledo, F \& et al, 2008). Existen opiniones que afirma, por ejemplo en Chile, que las normas que regulan actualmente el delito flagrante deben ser objeto de una reforma legal, debido a que se encuentran superadas ante la realidad (Reyes, A., 2009a). La aprehensión por delito flagrante se da con mayor frecuencia por contravenciones tanto de transito como delitos de violencia intrafamiliar; se hace más que necesaria la elaboración de un ensayo científico jurídico sobre la aprehensión del sospechoso en delito flagrante(Gómez, J., 2009). 
Lograr un desarrollo del tema redunda en la protección de los derechos de los procesados y las víctimas, ha de ser autónoma y no se puede inferir que es un deber o una obligación como comúnmente lo asocian la mayoría de las personas (Amaya, $\mathrm{H}$, 2009). La detención constitucional de flagrancia faculta a la policía en función a detener a una persona cuando se manifiesta los estados de flagrancia que prevé la nueva ley procesal. Ello exige un análisis inmediato de la autoridad policial. (Arcibia, García, \& et al, 2011).

Lo antes señalado, permite establecer que nuestra actual legislación ha flexibilizado la exigencia de la inmediación (temporal y personal) para establecer los supuestos de flagrancia, haciéndolos extensivos a supuestos que no constituyen propiamente supuestos de flagrancia.

El proceso inmediato permite simplificar, descongestionar y acelerar las etapas del proceso penal, en aquellos casos en que el fiscal no requiere de mayor información para tener la convicción de la existencia del delito y la responsabilidad del autor y se encuentra expedito para formular acusación. En nuestro ordenamiento, el proceso inmediato tiene en cierto modo su antecedente más directo en la Ley $\mathrm{N}^{\circ}$ 28122 del 16 de diciembre del 2003. En la propuesta, no se precisa la existencia de una, siquiera leve, fase de investigación formal, sino que simplemente en base a lo actuado preliminarmente el fiscal formula su requerimiento para pasar a juicio oral (Neyra, J., 2010). El proceso inmediato, se erige como una de las alternativas de celeridad procesal. Es uno de los procesos especiales que, bajo ciertas condiciones se aparta de la amplitud de trámite del proceso común(Aladino, T., 2009). Es un proceso especial que, en favor de la celeridad procesal, obvia la fase de investigación preparatoria propiamente dicha y la etapa intermedia cuando se presentan determinados supuestos (Ore, A., 2016). Por otra parte, el delito flagrante es el que encierra en sí la prueba de su realización, por lo que la flagrancia es la percepción sensorial directa del hecho delictivo. La finalidad de la flagrancia es impedir que el delito sea llevado a consecuencias ulteriores(Reátegui, J. \& et al, 2016). Se reconoce tres tipos de flagrancia: 1 . Flagrancia estricta: 2. Cuasiflagrancia: 3. Flagrancia presunta asi como también se considera la existencia de la flagrancia virtual. En el concepto clásico de flagrancia se requiere que el agente esté en el lugar de los hechos (inmediatez personal) y al momento preciso de los hechos o inmediatamente después (inmediatez temporal) (Vasquez, M., 2016). La flagrancia delictiva requiere inmediatez personal, inmediatez temporal y necesidad urgente. El autor al ser descubierto, o cuando es perseguido y capturado inmediatamente de haber realizado el acto punible (San Martín Castro, C, 2015). Aquellos actos realizados inmediatamente después de la consumación del delito, deben ser igualmente incluidos en la flagrancia(Meini, I., 2006). Las fases del proceso inmediato para tal fin, son Audiencia Única de incoación y Audiencia Única de Juicio. La ley $\mathrm{N}^{\mathrm{a}} 29659$ del 25 de agosto del 2010, que modificó el artículo 259 del CPP del 2004, acoge la concepción moderna de lo descrito.

\section{OBJETIVOS}

a) Conocer si con la aplicación del proceso inmediato en los casos de flagrancia se simplifican los actos procedimentales y de investigación que corroboren la existencia del delito y la responsabilidad penal del imputado.

b) Establecer si se otorga mayor celeridad a los procesos penales al impedir que transiten por todas las etapas del proceso penal.

c) Establecer si se cumple con satisfacer oportunamente el daño sufrido por las víctimas, así como las expectativas ciudadanas.

d) Establecer en qué medida se aplica el proceso inmediato en los casos de flagrancia en el Perú.

\section{METODOLOGÍA}

Diseño de investigación aplicada, porque está orientada en la aplicación de los conocimientos a la solución de un problema, pues confronta la teoria con la realidad(Hernández, R. \& et al, 2010). Tipo no experimental (ex post facto), de corte transversal referida al período 2016. También es un tipo de Investigación Socio Jurídica por que se estudian los hechos y relaciones de orden social reguladas por normas jurídicas. Se identificaron 50606 casos de flagrancia en el Perú. De esto se seleccionó una muestra probabilística de 212 profesionales entre jueces, fiscales penales y abogados que llevan casos penales a los cuales se les aplicó los instrumentos de recolección de información en forma personalizada

\section{RESULTADOS}

En el período de aplicación del proceso inmediato en los casos de flagrancia en el Perú, de un total de 50 606 procesos, el $54,19 \%$ correspondieron al delito de Omisión a la Asistencia Familiar, el $25,85 \%$ al delito de peligro común, el $7,06 \%$ al delito de hurto, el $3,49 \%$ al delito de robo, el $1,36 \%$ al delito de violencia y resistencia a la autoridad, y, el $8,05 \%$ al otro tipo de delitos.

El $90 \%$ de los jueces, $95 \%$ de los fiscales y $78 \%$ de los abogados señalan que la aplicación del proceso inmediato en los casos de flagrancia permite la simplificación del proceso penal (Tabla 1). 
Tabla 1: Aplicación del proceso inmediato respecto a la simplificación del proceso penal.

\begin{tabular}{lrrrrrr}
\hline & \multicolumn{6}{c}{ Muestra } \\
\cline { 2 - 7 } & Jueces & \multicolumn{3}{c}{ Fiscales } & Abogados \\
\cline { 2 - 7 } & $\mathrm{N}^{\circ}$ & $\%$ & $\mathrm{~N}^{\circ}$ & $\%$ & $\mathrm{~N}^{\circ}$ & $\%$ \\
\cline { 2 - 7 } $\mathbf{S i}$ & 19 & 90 & 20 & 95 & 165 & 78 \\
No & 2 & 10 & 1 & 5 & 35 & 17 \\
No sabe/No opina & 0 & 0 & 0 & 0 & 12 & 6 \\
\hline TOTAL & 21 & 100 & 21 & 100 & 212 & 100 \\
\hline
\end{tabular}

El $90 \%$ de los jueces, $83 \%$ de los fiscales y $74 \%$ de los abogados señalan que la aplicación del proceso inmediato en los casos de flagrancia permite la simplificación de la corroboración de la existencia del delito y la responsabilidad del imputado (tabla 2 ).

Tabla 2: Aplicación del proceso inmediato en los casos de flagrancia y la simplificación de la corroboración de la existencia del delito y la responsabilidad del imputado.

\begin{tabular}{lrrrrrr}
\hline \multirow{2}{*}{ Respuesta } & \multicolumn{6}{c}{ Muestra } \\
\cline { 2 - 7 } & \multicolumn{2}{c}{ Jueces } & \multicolumn{7}{c}{ Fiscales } & \multicolumn{2}{c}{ Abogados } \\
\cline { 2 - 7 } & $\mathrm{N}^{\circ}$ & $\%$ & $\mathrm{~N}^{\circ}$ & $\%$ & $\mathrm{~N}^{\circ}$ & $\%$ \\
\cline { 2 - 7 } Si & 19 & 90 & 34 & 83 & 156 & 74 \\
No & 2 & 10 & 7 & 17 & 44 & 20 \\
No sabe/No opina & 0 & 0 & 0 & 0 & 12 & 6 \\
TOTAL & 21 & 100 & 41 & 100 & 212 & 100 \\
\hline
\end{tabular}

El $90 \%$ de los jueces, $88 \%$ de los fiscales y $83 \%$ de los abogados señalan que la aplicación del proceso inmediato en los casos de flagrancia permite la eliminación del procedimiento rutinario e innecesario del proceso (Tabla 03)

Tabla 3: Aplicación del proceso inmediato y la eliminación del procedimiento rutinario

\begin{tabular}{lrrrrrr}
\hline Respuesta & \multicolumn{7}{c}{ Muestra } \\
\cline { 2 - 7 } & \multicolumn{1}{c}{ Jueces } & \multicolumn{3}{c}{ Fiscales } & Abogados \\
\cline { 2 - 7 } & $\mathrm{N}^{\circ}$ & $\%$ & $\mathrm{~N}^{\circ}$ & $\%$ & $\mathrm{~N}^{\circ}$ & $\%$ \\
\cline { 2 - 7 } $\mathrm{Si}$ & 19 & 90 & 36 & 88 & 175 & 83 \\
No & 2 & 10 & 5 & 12 & 28 & 13 \\
No sabe/No opina & 0 & 0 & 0 & 0 & 9 & 4 \\
TOTAL & 21 & 100 & 41 & 100 & 212 & 100 \\
\hline
\end{tabular}

El $86 \%$ de los jueces, $78 \%$ de los fiscales y el $74 \%$ de los abogados encuestados señalan que la aplicación del proceso inmediato en los casos de flagrancia impide que los procesos penales transiten por todas las etapas del proceso penal (Tabla 4).

Tabla 4: Aplicación del proceso inmediato y el impedimento del tránsito de los procesos penales por todas las etapas del proceso penal.

\begin{tabular}{lrrrrrr}
\hline Respuesta & \multicolumn{7}{c}{ Muestra } \\
\cline { 2 - 7 } & \multicolumn{2}{c}{ Jueces } & \multicolumn{3}{c}{ Fiscales } & \multicolumn{2}{c}{ Abogados } \\
\cline { 2 - 7 } & $\mathrm{N}^{\circ}$ & $\%$ & $\mathrm{~N}^{\circ}$ & $\%$ & $\mathrm{~N}^{\circ}$ & $\%$ \\
\cline { 2 - 7 } Si & 18 & 86 & 32 & 78 & 156 & 74 \\
No & 2 & 10 & 9 & 22 & 45 & 21 \\
No sabe/No opina & 1 & 4 & 0 & 0 & 11 & 5 \\
\cline { 2 - 7 } TOTAL & 21 & 100 & 41 & 100 & 212 & 100 \\
\hline
\end{tabular}

El $100 \%$ de los jueces, $90 \%$ de los fiscales y el $78 \%$ de los abogados encuestados señalan que la aplicación del proceso inmediato en los casos de flagrancia otorga mayor celeridad a los procesos penales (Tabla 5). 
Tabla 5: Aplicación del proceso inmediato y la celeridad de los procesos penales.

\begin{tabular}{lrrrrrr}
\hline Respuesta & \multicolumn{7}{c}{ Muestra } \\
\cline { 2 - 7 } & \multicolumn{1}{c}{ Jueces } & \multicolumn{2}{c}{ Fiscales } & \multicolumn{2}{c}{ Abogados } \\
\cline { 2 - 7 } & $N^{\circ}$ & $\%$ & $N^{\circ}$ & $\%$ & $N^{\circ}$ & $\%$ \\
\cline { 2 - 7 } Si & 21 & 100 & 37 & 90 & 165 & 78 \\
No & 0 & 0 & 3 & 7 & 35 & 17 \\
No sabe/No opina & 0 & 0 & 1 & 3 & 12 & 5 \\
TOTAL & 21 & 100 & 41 & 100 & 212 & 100 \\
\hline
\end{tabular}

El $100 \%$ de los jueces, $88 \%$ de los fiscales y $79 \%$ de los abogados señalan que la aplicación del proceso inmediato en los casos de flagrancia permite sancionar los hechos delictivos facilitando la intervención del Estado para la rápida conclusión del proceso penal (Tabla 06).

Tabla 6: Aplicación del proceso inmediato y la sanción de los hechos delictivos

\begin{tabular}{lrrrrrr}
\hline & \multicolumn{6}{c}{ Muestra } \\
\cline { 2 - 7 } & \multicolumn{2}{c}{ Jueces } & \multicolumn{3}{c}{ Fiscales } & Abogados \\
\cline { 2 - 7 } & $\mathrm{N}^{\circ}$ & $\%$ & $\mathrm{~N}$ & $\%$ & $\mathrm{~N}^{\circ}$ & $\%$ \\
\cline { 2 - 7 } Si & 21 & 100 & 36 & 88 & 168 & 79 \\
No & 0 & 0 & 5 & 12 & 32 & 15 \\
No sabe/No opina & 0 & 0 & 0 & 0 & 12 & 6 \\
TOTAL & 21 & 100 & 41 & 100 & 212 & 100 \\
\hline
\end{tabular}

El $86 \%$ de los jueces, $85 \%$ de los fiscales y $87 \%$ de los abogados encuestados señalan que la aplicación del proceso inmediato en los casos de flagrancia permite alcanzar la justicia oportuna en los casos aplicados (tabla 7).

Tabla 7: La aplicación del proceso inmediato y la justicia oportuna.

\begin{tabular}{lrrrrrr}
\hline Respuesta & \multicolumn{7}{c}{ Muestra } \\
\cline { 2 - 7 } & Jueces & \multicolumn{3}{c}{ Fiscales } & Abogados \\
& $N^{\circ}$ & $\%$ & $N^{\circ}$ & $\%$ & $N^{\circ}$ & $\%$ \\
Si & 18 & 86 & 35 & 85 & 184 & 87 \\
No & 2 & 10 & 5 & 12 & 21 & 10 \\
No sabe/No opina & 1 & 4 & 1 & 3 & 7 & 3 \\
TOTAL & 21 & 100 & 41 & 100 & 212 & 100 \\
\hline
\end{tabular}

El $71 \%$ de los jueces, $80 \%$ de los fiscales y $81 \%$ de los abogados encuestados señalan que se cumple con aplicar la finalidad del proceso inmediato en los casos de flagrancia (tabla 8).

Tabla 8: Cumplimiento de aplicación del proceso inmediato

\begin{tabular}{lrrrrrr}
\hline Respuesta & \multicolumn{7}{c}{ Muestra } \\
\cline { 2 - 7 } & Jueces & \multicolumn{1}{c}{ Fiscales } & \multicolumn{2}{c}{ Abogados } \\
\cline { 2 - 7 } & $N^{\circ}$ & $\%$ & $N^{\circ}$ & $\%$ & $N^{\circ}$ & $\%$ \\
Si & 15 & 71 & 33 & 80 & 172 & 81 \\
No & 5 & 24 & 7 & 17 & 32 & 15 \\
No sabe/No opina & 1 & 5 & 1 & 3 & 8 & 4 \\
TOTAL & 21 & 100 & 41 & 100 & 212 & 100 \\
\hline
\end{tabular}

El $19 \%$ de los jueces, $17 \%$ de los fiscales y $4 \%$ de los abogados señalan que los casos incoados de flagrancia por proceso inmediato en el Perú son altos (Tabla 9). 
Tabla 9: Casos incoados en flagrancia por proceso inmediato en el Perú.

\begin{tabular}{lrrrrrr}
\hline Respuesta & \multicolumn{7}{c}{ Muestra } \\
\cline { 2 - 7 } & \multicolumn{1}{c}{ Jueces } & \multicolumn{3}{c}{ Fiscales } & Abogados \\
\cline { 2 - 7 } & $\mathrm{N}^{\mathbf{0}}$ & $\%$ & $\mathrm{~N}^{\circ}$ & $\%$ & $\mathrm{~N}^{\circ}$ & $\%$ \\
\cline { 2 - 7 } Alto & 4 & 19 & 7 & 17 & 8 & 4 \\
Medio & 6 & 29 & 13 & 32 & 30 & 14 \\
Bajo & 11 & 52 & 21 & 51 & 174 & 82 \\
TOTAL & 21 & 100 & 41 & 100 & 212 & 100 \\
\hline
\end{tabular}

El $71 \%$ de los jueces, $88 \%$ de los fiscales y $89 \%$ de los abogados señalan que se debe modificar los plazos y mejorar los recursos para garantizar la eficiencia del proceso inmediato en los casos de flagrancia (tabla 10).

Tabla 10: Modificación de los plazos para garantizar la eficiencia del proceso inmediato en los casos de flagrancia.

\begin{tabular}{lrrrrrr}
\hline Respuesta & \multicolumn{7}{c}{ Muestra } \\
\cline { 2 - 7 } & \multicolumn{1}{c}{ Jueces } & \multicolumn{3}{c}{ Fiscales } & \multicolumn{3}{c}{ Abogados } \\
\cline { 2 - 7 } & $\mathrm{N}^{\mathbf{0}}$ & $\%$ & $\mathrm{~N}^{\circ}$ & $\%$ & $\mathrm{~N}^{\circ}$ & $\%$ \\
\cline { 2 - 7 } Si & 15 & 71 & 36 & 88 & 189 & 89 \\
No & 4 & 19 & 3 & 7 & 14 & 7 \\
No sabe/No opina & 2 & 10 & 2 & 5 & 9 & 4 \\
TOTAL & 21 & 100 & 41 & 100 & 212 & 100 \\
\hline
\end{tabular}

Los resultados de la entrevista aplicada a los magistrados encargados de los Juzgados Penales se rescató lo siguiente:

Respecto a la simplificación del proceso penal, con la aplicación del proceso inmediato en los delitos de flagrancia se han resuelto varios casos, disminuyendo las personas procesadas sin condena, así como la reducción de los plazos para la emisión por parte de las autoridades jurisdiccionales de las resoluciones pero hay que advertir los riesgos que ello podría traer consigo, como en su aplicación se dé una mala praxis por parte de los operadores de justicia o que la pena sea desproporcional o se violen las garantías procesales que les asiste a toda persona sometida a un proceso. Respecto a la celeridad del proceso penal. La simplificación procesal prevista en el Nuevo Código Procesal Penal ha sido positiva demás que en el análisis de si ha permitido alcanzar la justicia oportuna es menester señalar que tanto la flagrancia como el proceso inmediato generan una respuesta de manera alentadora al pedido o clamor de una sociedad en busca de justicia, aportando así a la seguridad ciudadana, sin embargo no aplicarse dicha medida como solución populista del momento que calme la inseguridad ciudadana. En lo referente al cumplimiento de la finalidad del proceso inmediato en la mayoría de casos de flagrancia se señala que si bien su aplicación refleja una situación aliciente, sin embargo también es de advertir que la emisión que una sentencia (absolutoria o condenatoria) rápida y efectiva no constituirian aparentemente más que una ilusión, cuya única consecuencia va en contra del propio individuo. Hay que tener presente que una simplificación procesal no sólo confluye que tan bien redactada se encuentre la norma, sino también que tan capacitados se encuentran los operadores de justicia para aplicarla. El impacto finalmente es positivo de por si para la sociedad, pero también a todo ello es importante no dejar de lado lo señalado en los puntos precedentes. Se debe tener en cuenta que no todo delito de flagrancia es de simple o sencilla resolución. Se debe considerar no dejar de lado las garantías procesales que gobiernan todo proceso asi como una mayor capacitación al personal jurisdiccional y a todos los magistrados con el respeto irrestricto a la Constitución y la ley.

\section{DISCUSIÓN}

El impacto de la aplicación del proceso inmediato en los casos de flagrancia en el Perú 2016 confirma que la aplicación del proceso inmediato en los casos de flagrancia simplifica los actos procedimentales y de investigación que corroboren la existencia del delito y la responsabilidad penal del imputado, al hallarse que otorga mayor celeridad a los procesos penales satisface oportunamente el daño sufrido por las víctimas. En el Perú se aplica el proceso inmediato en los casos de flagrancia en menor medida. Estos resultados guardan relación con el estudio de Castejón (Castejón, M., 2009) . El Procedimiento de flagrancia en el derecho procesal penal Venezolano. Universidad Católica Andrés Bello, concluye lo 
siguiente: El efecto suspensivo establecido en el artículo 374 del COPP, que paradójicamente fue concebido para coadyuvar en la solución de la grave problemática que se presentó al comienzo de la vigencia de esta importante Ley, con las libertades otorgadas en casos de detenciones in fraganti, en los que no se calificaba la flagrancia, constituye la más grave falla que presenta el procedimiento abreviado, por cuando como quedó expuesto, atenta no sólo contra normas de orden legal, sino que afecta también una norma constitucional, situación que si bien puede ser resuelta por los tribunales de conformidad con las previsiones que consagran los controles difusos y concentrado de la Constitución, no tiene justificación en una ley de orden garantista como el COPP, por lo que se considera que no obstante la mejor interpretación jurisprudencial que se haga de dicha norma, debe ser incluida para ser corregida en una futura ley procesal penal. Asimismo, los resultados del presente estudio también guardan relación con el estudio de: Reyes,(Reyes, A., 2009) . Respecto al Delito flagrante y sus implicancias en el proceso penal, la Universidad Austral de Chile, señala lo siguiente: Las normas que regulan actualmente el delito flagrante deben ser objeto de una reforma legal, debido a que se encuentran superadas ante la realidad, no han evolucionado, y esta situación puede ser el inicio para una serie de críticas al actual sistema procesal penal. El sistema procesal penal actualmente contiene los mecanismos de protección adecuados frente a un actuar que sobrepase las actuales normas que regulan la detención por delito flagrante, principalmente a través de la audiencia de control de la detención, complementado esto por la fundamental circunstancia que el imputado es asistido desde la primera actuación del procedimiento por un abogado, quien está facultado para ejercer todos los derechos que le confiere el Código Procesal Penal, a diferencia de lo que ocurría con el Código de Procedimiento Penal. La actual regulación del delito flagrante constituye una desventaja para el actuar policial, por cuanto puede ser dejada sin efecto la detención efectuada en la primera audiencia judicial, lo que redunda en una pérdida de confianza en su actuar. Sugerimos que el Poder Legislativo debe coordinar con el Poder Judicial a efecto que se establezca un marco normativo que permita mejorar el sistema de justicia, establecer una política criminal interdisciplinaria, donde El Instituto Nacional Penitenciario conjuntamente con el Poder Judicial, puedan coordinar con los Gobiernos Regionales y Locales, a fin que las sanciones que dicte el Poder Judicial, en los procesos inmediatos, se cumplan mediante la prestación de servicios a la comunidad, dejándose de lado las penas suspendidas. Se debe considerar la implementación de programas de capacitación para abogados privados; de modo que logren realizar una defensa técnica conforme a los intereses de sus representados. El Congreso de la República debe modificar las normas sobre el proceso inmediato, el cual debe ser solamente para los delitos simples y cuya pena no exceda a los seis años.

Podemos concluir que la aplicación del proceso inmediato en los casos de flagrancia simplifica los actos procedimentales y de investigación que corroboren la existencia del delito y la responsabilidad penal del imputado, otorga mayor celeridad a los procesos y satisface oportunamente las expectativas ciudadanas así como el daño sufrido por las víctimas.

\section{BIBLIOGRAFÍA}

Aladino, T. (2009). El Código Procesal Penal. Lima: Iurista Editores, p.829.

Amaya, H. (2009). La Captura en flagrancia: una potestad del oficial de cumplimiento de la ley con implicaciones en materia procesal penal. Universidad Regional Autónoma de los Andes Uniandes - Ibarra.

Arcibia, García, \& et al. (2011). La Flagrancia en el Nuevo Procesal Penal. Universidad de San Martin de Porres.

Castejón, M. (2009). El Procedimiento de flagrancia en el derecho procesal penal Venezolano. Universidad Católica Andrés Bello.

Gómez, J. (2009). La aprehensión en delito flagrante y sus efectos jurídicos en la Legislación Ecuatoriana. Universidad Regional Autónoma De Los Andes Uniandes Ibarra.

Hernández, R., \& et al. (2010). Metodología de la Investigación. (1ra edición). Colombia: Mc Graw-Hill.

Meini, I. (2006). Procedencia y requisitos de la detención. En: La Constitución comentada. Tomo 1, Lima: Gaceta Jurídica, p. 294.

Neyra, J. (2010). Manuel del Nuevo Código Procesal Penal \& Litigación oral. Lima: Idemsa,pp.431-434.

Ore, A. (2016). Estudio Introductorio del Proceso Inmediato. Lima: Gaceta Jurídica S.A.,p.7.

Reátegui, J., \& et al. (2016). El Proceso Penal Inmediato en casos de flagrancia delictiva. Lima: Legales Ediciones, p.58-62.

Rebolledo, F, \& et al. (2008). La Flagrancia: ¿Hipótesis Indiscutible?. Escuela de Derecho Universidad Católica de Temuco.

Reyes, A. (2009a). El Delito flagrante y sus implicancias en el proceso penal. Universidad Austral de Chile.

Reyes, A. (2009b). El Delito flagrante y sus implicancias en el proceso penal. Universidad Austral de Chile.

San Martín Castro, C. (2015). Derecho Procesal Penal. Lecciones.Lima: Fondo editorial del INPECCP, p.448.

Vasquez, M. (2016). La incoación del Nuevo Proceso Inmediato, en el Nuevo

Proceso Penal Inmediato. Lima: Gaceta Jurídica.p.9.

Recibidos: 06 / 04 / 16

Aceptado para publicación: 9 / 6 / 17 